University of Nebraska - Lincoln

DigitalCommons@University of Nebraska - Lincoln

Faculty Publications from the Department of Electrical \& Computer Engineering, Department Electrical and Computer Engineering

2011

\title{
THz dielectric anisotropy of metal slanted columnar thin films
}

Tino Hofmann

University of Nebraska-Lincoln, thofmann4@unl.edu

D. Schmidt

University of Nebraska-Lincoln

A. Boosalis

University of Nebraska-Lincoln

P. Kuhne

University of Nebraska-Lincoln

Ralph Skomski

University of Nebraska-Lincoln, rskomski2@unl.edu

See next page for additional authors

Follow this and additional works at: https://digitalcommons.unl.edu/electricalengineeringfacpub

Part of the Electrical and Computer Engineering Commons

Hofmann, Tino; Schmidt, D.; Boosalis, A.; Kuhne, P.; Skomski, Ralph; Herzinger, C. M.; Woollam, John A.; Schubert, Mathias; and Schubert, Eva, "THz dielectric anisotropy of metal slanted columnar thin films" (2011). Faculty Publications from the Department of Electrical and Computer Engineering. 170. https://digitalcommons.unl.edu/electricalengineeringfacpub/170

This Article is brought to you for free and open access by the Electrical \& Computer Engineering, Department of at DigitalCommons@University of Nebraska - Lincoln. It has been accepted for inclusion in Faculty Publications from the Department of Electrical and Computer Engineering by an authorized administrator of DigitalCommons@University of Nebraska - Lincoln. 


\section{Authors}

Tino Hofmann, D. Schmidt, A. Boosalis, P. Kuhne, Ralph Skomski, C. M. Herzinger, John A. Woollam, Mathias Schubert, and Eva Schubert 


\title{
THz dielectric anisotropy of metal slanted columnar thin films
}

\author{
T. Hofmann, ${ }^{1, a)}$ D. Schmidt, ${ }^{1}$ A. Boosalis, ${ }^{1}$ P. Kühne, ${ }^{1}$ R. Skomski, ${ }^{2}$ C. M. Herzinger, ${ }^{3}$ \\ J. A. Woollam, ${ }^{3}$ M. Schubert, ${ }^{1}$ and E. Schubert ${ }^{1}$ \\ ${ }^{1}$ Department of Electrical Engineering and Nebraska Center for Materials and Nanoscience, \\ University of Nebraska-Lincoln, Lincoln, Nebraska 68588-0511, USA \\ ${ }^{2}$ Department of Physics and Astronomy and Nebraska Center for Materials and Nanoscience, \\ University of Nebraska-Lincoln, Lincoln, Nebraska 68588-0511, USA \\ ${ }^{3}$ J.A. Woollam Co. Inc., 645 M Street, Suite 102, Lincoln, Nebraska 68508-2243, USA
}

(Received 29 April 2011; accepted 31 July 2011; published online 23 August 2011)

\begin{abstract}
The anisotropic optical dielectric functions of a metal (cobalt) slanted columnar thin film deposited by electron-beam glancing angle deposition are reported for the terahertz $(\mathrm{THz})$ frequency domain using generalized spectroscopic ellipsometry. We employ a simple effective medium dielectric function homogenization approach to describe the observed optical response. The approach describes isolated, electrically conductive columns which render the thin film biaxial (orthorhombic). Our findings suggest controlled variability of dielectric polarizability and anisotropy in the $\mathrm{THz}$ spectral range by choice of geometry, material, and structure. (C) 2011 American Institute of Physics. [doi:10.1063/1.3626846]
\end{abstract}

Sculptured thin films (STFs) present an interesting class of self-organized, artificially made materials with threedimensional, highly spatially coherent arrangements of nanostructures. The intrinsic building block is formed of columns with a controllable inclination angle towards the substrate surface (Fig. 1). Geometry, dimension, and fabrication material are accessible design parameters, and STFs can be tailored to obtain varying physical properties. STF properties such as reflectance, transmittance, and conductance may differ significantly from the constituent material's bulk form. ${ }^{1-3}$ Contemporary interest in materials for terahertz $(\mathrm{THz})$ electronic, optoelectronic, and optical applications is redrawing attention to STFs that may enable designed optical properties for the $\mathrm{THz}$ frequency region. In this frequency range, existing materials are either purely dielectric and transparent or electrically conductive and highly absorbing. STFs prepared from electrically conductive materials offer the interesting opportunity to design transparent materials with very large dielectric polarizabilities. The strong polarizability originates from the highly coherent electrically insulated arrays of subwavelength dipole antennas. Coupling between adjacent dipoles leads to directionally dependent electromagnetic response, observable as an optical anisotropy. Few information is available on the optical properties and anisotropic refraction and extinction coefficients of metal STFs. Recently, we have demonstrated that generalized spectroscopic ellipsometry (GSE) in the visible spectral range serves as an ideal tool for the determination of the anisotropic dielectric properties of STFs. ${ }^{4-6}$ No information seems to exist on metal STF optical properties in the THz region.

In this work, we determine the anisotropic optical properties of a Co STF sample in the THz spectral range. THzGSE which has become available very recently is employed here. $^{7-12}$ We demonstrate that the anisotropic Bruggeman effective medium theory (AB-EMA) can be used in order to

\footnotetext{
${ }^{\text {a) }}$ Author to whom correspondence should be addressed. Electronic address: thofmann@engr.unl.edu.
}

accurately describe the anisotropic optical response of the STFs. Complementary angle-resolved GSE measurements in the spectral range from 400 to $1680 \mathrm{~nm}$ and scanning electron microcopy (SEM) were performed in order to determine the thickness of the STF and AB-EMA parameters for comparison.

The sample studied here is composed of a $450 \mathrm{~nm}$ thick Co STF grown by electron-beam glancing angle deposition in a customized ultrahigh vacuum chamber onto a low phosphorous-doped $n$-type (001) silicon substrate. The deposition was carried out at an angle of $85^{\circ}$ between the incident particle flux direction and the substrate normal. Further details about the growth process are omitted here and the interested reader is referred to Ref. 4. The slanting angle of the Co nanocolumns is $65^{\circ}$. A high-resolution field-emission SEM edge view image of the sample is shown in Figure 1. The THz-GSE measurements were carried out in the spectral range from 0.65 to $1.00 \mathrm{THz}$ with a resolution of $1 \mathrm{GHz}$ using a custom-built frequency-domain $\mathrm{THz}$ ellipsometer, which has been described in detail in Refs. 8 and 11. Experimental THz-GSE data, which are represented here using the Mueller matrix formalism, ${ }^{13}$ were obtained at two different angles of incidence $\Phi_{\mathrm{a}}=55^{\circ}$ and $65^{\circ}$ and for three different

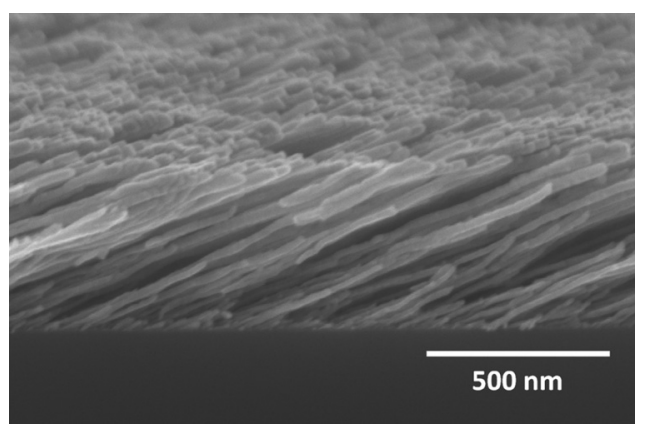

FIG. 1. SEM micrograph (sample tilted $150^{\circ}$ ) of the investigated slanted Co nanocolumns deposited by glancing angle deposition onto a silicon substrate. The slanting angle is $65^{\circ}$. 
in-plane sample rotation angles $\varphi=90^{\circ}, 135^{\circ}$, and $180^{\circ}$ and for which the columnar slanting plane is oriented parallel, oblique $\left(45^{\circ}\right)$, and perpendicular to the plane of incidence, respectively.

The experimental THz-GSE data sets were analyzed using a stratified layer model calculation where all model calculated data were matched simultaneously as closely as possible to the experimental THz-GSE data sets by varying relevant physical model parameters (best-model). ${ }^{14}$ The anisotropic dielectric functions of the Co STF sample is described here by an anisotropic Bruggeman effective medium approximation. The Bruggeman formalism describes the homogenization for randomly oriented elliptical inclusions in a homogeneous host medium. This formalism can be generalized for the case of highly oriented elliptical inclusions. ${ }^{15-18}$ For highly spatially coherent oriented elliptical inclusions, the effective dielectric function tensor is described by the three major components $\varepsilon_{a}, \varepsilon_{b}$, and $\varepsilon_{c}$ along the major axes $a, b$, and $c$ of an orthorhombic system. $\varepsilon_{j}$ with $j=a, b, c$ are given in implicit form by ${ }^{19}$

$$
\varepsilon_{j}=\varepsilon_{\mathrm{m}}+\frac{f\left(\varepsilon_{\mathrm{i}}-\varepsilon_{\mathrm{m}}\right) \varepsilon_{j}}{\varepsilon_{j}+L_{j}\left(\varepsilon_{\mathrm{i}}-\varepsilon_{j}\right)},
$$

where the dielectric permittivity and volume fraction of the nanocolumnar inclusions are denoted by $\varepsilon_{\mathrm{i}}$ and $f$, respectively. $\varepsilon_{\mathrm{m}}$ is the permittivity of the host medium, i.e., $\varepsilon_{\mathrm{m}}=1$ for the sample under investigation here. $L_{j}$ are the depolarization factors of the elliptical inclusions along the major polarizability axes. Regardless of ellipsoid shape, the sum of the three depolarization factors satisfies $L_{a}+L_{b}+L_{c}=1{ }^{18}$ In the $\mathrm{THz}$ spectral range, the dielectric function of the $\mathrm{Co}$ (metal) nanocolumnar inclusions $\varepsilon_{\mathrm{i}}$ is described by the classical Drude formalism ${ }^{20,21}$

$$
\varepsilon_{\mathrm{i}}(\omega)=\varepsilon_{\infty}-\varepsilon_{\infty} \frac{\omega_{\mathrm{p}}^{2}}{\omega(\omega+i / \tau)},
$$

where $\omega_{p}$ is the screened plasma frequency and $\tau$ is the average energy-independent scattering time. The DC conductivity $\sigma_{d c}$ is related to $\omega_{p}$ by $\sigma_{d c}=\varepsilon_{\infty} \tilde{\varepsilon}_{0} \omega_{p}^{2} \tau$, where $\omega_{p}^{2}=N q^{2} /\left(\varepsilon_{\infty} \tilde{\varepsilon}_{0} m\right)$ and $\mu$ is the mobility given by $\mu=q \tau / m$, $\tilde{\varepsilon}_{0}$ is the vacuum permittivity, $N$ is the free charge carrier concentration, and $q$ denotes the charge. The high frequency dielectric constant and the effective mass of the free charge carriers are denoted by $\varepsilon_{\infty}$ and $m$, respectively.

For best-match model calculations, a three phase model consisting of a low doped $n$-type silicon substrate/Co STF layer/ambient was implemented. Figure 2 depicts the nontrivial THz-GSE data $M_{12}, M_{21}$, and $M_{33}$ of the Co STF sample at an angle of incidence of $\Phi_{\mathrm{a}}=55^{\circ}$ for three different in-pane rotations $\varphi=90^{\circ}, 135^{\circ}$, and $180^{\circ}$. For comparison, the Mueller matrix spectra of the silicon substrate before Co STF deposition are plotted. The experimental (dotted lines) and best-model calculated (solid lines) data are found to be in very good agreement. The Mueller matrix spectra are dominated by Fabry-Pérot interference pattern originating from the entire sample. The frequency of the Fabry-Pérot interference pattern is determined by the layer thicknesses of all sample constituents, but it is dominated by the thickness

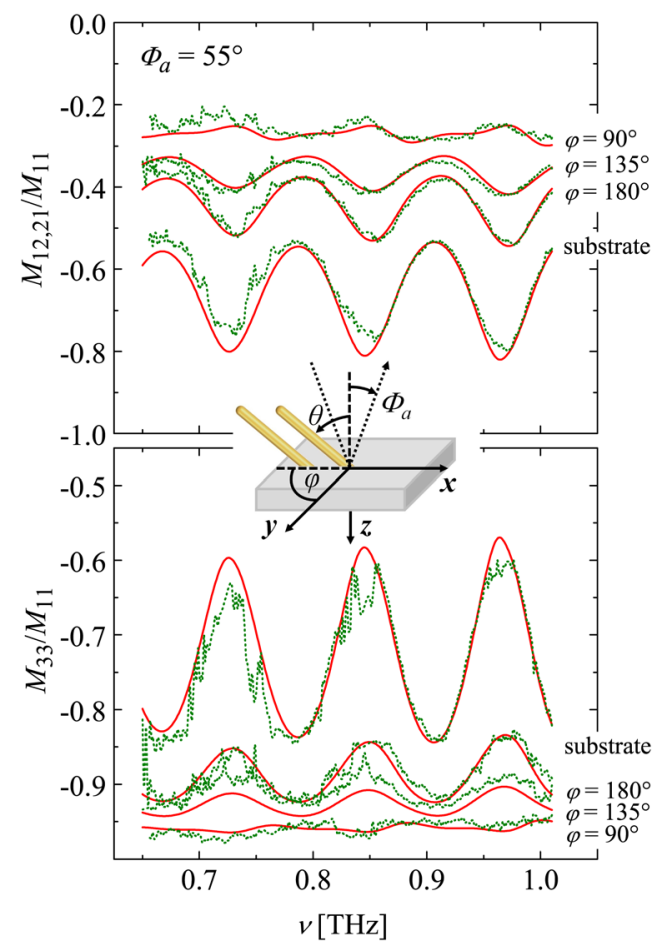

FIG. 2. (Color online) Experimental (dotted lines) and best-model calculated (solid lines) non-trivial Mueller matrix spectra (upper panel: $M_{12}$ and $M_{21}$, lower panel: $M_{33}$ ) for the Co STF sample for three different in-plane rotation angles $\varphi=90^{\circ}, 135^{\circ}$, and $180^{\circ}$. The spectra for the silicon substrate before Co STF deposition are shown for comparison.

of the substrate which is $378 \pm 1 \mu \mathrm{m}$. At the first glance, one can observe that the interference amplitude is reduced for the Co STF sample in comparison to the bare silicon substrate due to the presence of the nanocolumnar film. It can be seen in Fig. 2 that the Fabry-Pérot pattern observed for the Co STF sample change as a function of the in-plane rotation angle. With increasing $\varphi$, the orientation of the slanted nanocolumns changes from parallel to perpendicular to the plane of incidence and the damping effect decreases.

Figure 3 depicts the off-diagonal components of the Mueller matrix of the Co STF sample obtained at an angle of incidence of $\Phi_{\mathrm{a}}=55^{\circ}$ and $\varphi=135^{\circ} .{ }^{22}$ The anisotropy of the optical response of the nanocolumnar thin film is evident from the non-zero off-diagonal elements $M_{13}, M_{31}, M_{23}, M_{32}$

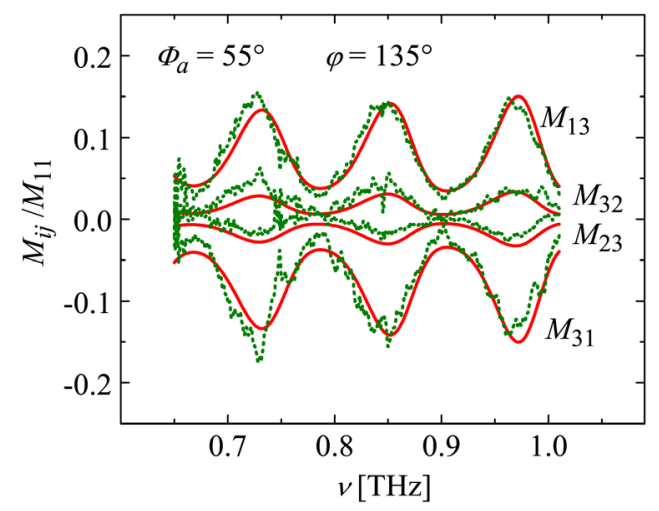

FIG. 3. (Color online) Experimental (dotted lines) and best-model calculated (solid lines) off-diagonal Mueller matrix spectra for the Co STF sample for an in-plane rotation angle $\varphi=135^{\circ}$. 
which would vanish for an isotropic sample. The best-model parameters for the AB-EMA depolarization factors are: $L_{a}=0.3333(1), L_{b}=0.3355(1)$, and $L_{c}=0.3312(1)$. The depolarization factors render the dipole shape at the location of the inclusion and are wavelength dependent. In the $\mathrm{THz}$ frequency range, the polarization fields are produced by thousands of neighboring nanocolumns. Probed at large wavelengths, factors $L_{j}$ render the shape of the induced polarization almost as a sphere, however, with subtle differences along and perpendicular to the columnar axis. These subtle differences are responsible for the anisotropic dielectric functions, which differ all drastically from the corresponding bulk (cobalt) dielectric properties. The best-model parameters for the STF thickness and volume fraction of the nanocolumns, $d=441(5) \mathrm{nm}$, and $f=33(1)$, respectively, are in good agreement with SEM data obtained from Figure 1 and GSE measurements in the NIR-VIS spectral range. ${ }^{23}$ As additional structural parameter, we also determined the slanting angle of the intrinsic Cartesian coordinate system of the polarizabilities $\varepsilon_{j}$, as described previously ${ }^{4-6}$ and which is identical with the physical slanting angle of the nanocolumns. For the Drude model parameters of the Co nanocolumns, we obtained $\rho=1.2(3) \times 10^{-5} \Omega \mathrm{cm}$ and $\tau=239(7)$ fs. We find that these parameters are in very good agreement to those obtained for a bulk-like $100 \mathrm{~nm}$ thick Co film on low doped silicon substrate which was analyzed in the same spectral range for comparison. The resistivity model parameter of the Co nanocolumns is approximately twice as large as reported for bulk Co samples. ${ }^{24}$ Overall, the agreement with bulk and thin film conductivity is exciting given the limitations of the approximate AB-EMA model approach. The best-model parameters for the silicon substrate are $N=1.08(4) \times 10^{15} \mathrm{~cm}^{-3}$ and $\mu=1375(111) \mathrm{cm}^{2} /(\mathrm{Vs})$ if an electron effective mass of $0.26 m_{0}$ (Ref. 25) is assumed. The anisotropic dielectric functions $\varepsilon_{j}$ and their coordinate system

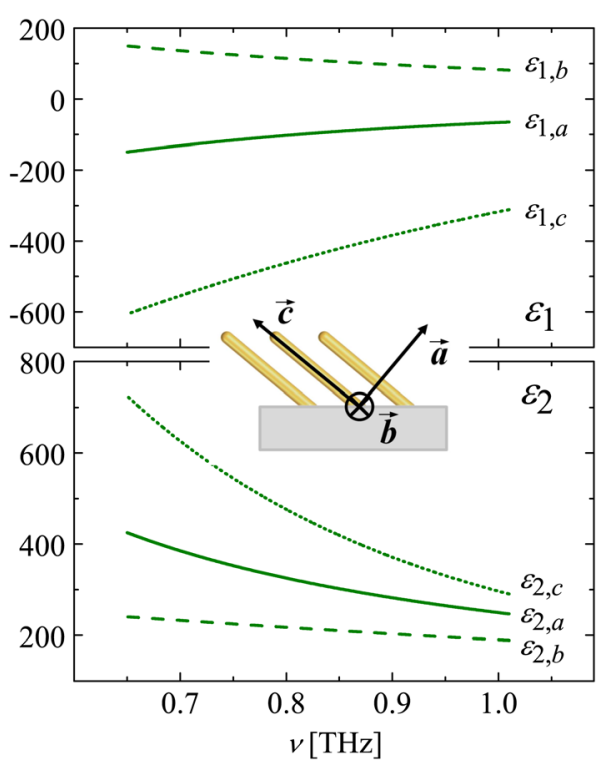

FIG. 4. (Color online) Real (upper panel: $\varepsilon_{1, a}, \varepsilon_{1, b}$, and $\varepsilon_{1, c}$ ) and imaginary (lower panel: $\varepsilon_{2, a}, \varepsilon_{2, b}$, and $\varepsilon_{2, c}$ ) parts of the $\mathrm{THz}$ orthorhombic dielectric functions obtained from the best-model calculation for the Co STF sample. The inset depicts the axes locations of the intrinsic Cartesian polarizability system. are depicted in Fig. 4. The anisotropy induced by the columnar film structure is very large. Note that while the dielectric polarizability (real part of $\varepsilon_{j}$ ) is larger than for most dielectric materials, the absorption (imaginary part of $\varepsilon_{j}$ ) is reduced by approximately two orders of magnitude compared with bulk or thin film Co. The AB-EMA model approach predicts upon slight modifications of Drude, fraction, and/or depolarization parameters that targeted optical properties of STF in the THz range can be achieved by variation of slanting angle, lateral column density, and material.

In summary, we have determined the anisotropic optical dielectric functions of a Co STF sample in the THz range and applied an anisotropic effective medium approach in order to identify and describe the orthorhombic dielectric functions of the STF.

The authors would like to acknowledge financial support from the Army Research Office (W911NF-09-C-0097), the National Science Foundation (MRSEC DMR-0820521, MRI DMR-0922937, DMR-0907475, ECCS-0846329, and EPS1004094), the University of Nebraska-Lincoln, and the J.A. Woollam Foundation.

${ }^{1}$ I. J. Hodgkinson and Q. H. Wu, Birefringent Thin Films and Polarizing Elements (World Scientific, Singapore, 1998).

${ }^{2}$ M. M. Hawkeye and M. J. Brett, J. Vac. Sci. Technol. A 25, 1317 (2007).

${ }^{3}$ C. Buzea, K. Kaminska, G. Beydaghyan, T. Brown, C. Elliott, C. Dean, and K. Robbie, J. Vac. Sci. Technol. B 23, 2545 (2005).

${ }^{4}$ D. Schmidt, A. C. Kjerstad, T. Hofmann, R. Skomski, E. Schubert, and M. Schubert, J. Appl. Phys. 105, 113508 (2009).

${ }^{5}$ D. Schmidt, B. Booso, T. Hofmann, E. Schubert, A. Sarangan, and M. Schubert, Appl. Phys. Lett. 94, 011914 (2009).

${ }^{6}$ D. Schmidt, B. Booso, T. Hofmann, E. Schubert, A. Sarangan, and M. Schubert, Opt. Lett. 34, 992 (2009).

${ }^{7}$ A. K. Azad, J. Han, and W. Zhang, Appl. Phys. Lett. 88, 021103 (2006).

${ }^{8}$ T. Hofmann, C. Herzinger, A. Boosalis, T. Tiwald, J. Woollam, and M. Schubert, Rev. Sci. Instrum. 81, 023101 (2010).

${ }^{9}$ N. Matsumoto, T. Hosokura, T. Nagashima, and M. Hangyo, Opt. Lett. 36, 265 (2011).

${ }^{10}$ T. Hofmann, A. Boosalis, P. Kühne, C. M. Herzinger, J. A. Woollam, D. K. Gaskill, J. L. Tedesco, and M. Schubert, Appl. Phys. Lett. 98, 041906 (2011).

${ }^{11}$ T. Hofmann, C. M. Herzinger, J. L. Tedesco, D. K. Gaskill, J. A. Woollam, and M. Schubert, Thin Solid Films 519, 2593 (2011).

${ }^{12}$ S. Schöche, J. Shi, A. Boosalis, P. Kühne, C. M. Herzinger, J. A. Woollam, W. J. Schaff, L. F. Eastman, M. Schubert, and T. Hofmann, Appl. Phys. Lett. 98, 092103 (2011).

${ }^{13}$ H. Fujiwara, Spectroscopic Ellipsometry (John Wiley \& Sons, New York, 2007).

${ }^{14}$ G. E. Jellison, Thin Solid Films 313-314, 33 (1998).

${ }^{15}$ D. Bruggeman, Ann Phys 24, 636 (1935).

${ }^{16}$ D. Aspnes, Thin Solid Films 89, 249 (1982).

${ }^{17}$ G. Smith, Opt. Commun. 71, 279 (1989).

${ }^{18}$ A. Sihvola, Electromagnetic Mixing Formulas and Applications (The Institution of Electrical Engineers, London, 1999).

${ }^{19}$ B. C. Bergner, T. A. Germer, and T. J. Suleski, J. Opt. Soc. Am. A 27, 1083 (2010).

${ }^{20} \mathrm{C}$. Pidgeon, in Handbook on Semiconductors, edited by M. Balkanski (North-Holland, Amsterdam, 1980).

${ }^{21}$ M. Schubert, Infrared Ellipsometry on Semiconductor Layer Structures: Phonons, Plasmons and Polaritons, Springer Tracts in Modern Physics Vol. 209 (Springer, Berlin, 2004).

${ }^{22}$ The employed THz ellipsometer gives access to the upper $3 \times 3$ block of the Mueller matrix only (Ref. 8).

${ }^{23}$ The measurements were carried out as described in Ref. 6. Details on the analysis are subject to a future publication.

${ }^{24}$ P. Pureur, J. V. Kunzler, W. H. Schreiner, and D. E. Brando, Phys. Status. Solidi A 70, 11 (1982).

${ }^{25}$ M. van Exter and D. Grischkowsky, Appl. Phys. Lett. 56, 1694 (1990). 